Acta Crystallographica Section E

Structure Reports

Online

ISSN 1600-5368

\section{(E)-2-(Cyclohexylmethylene)succinic acid}

\section{Wei Wang, ${ }^{a} *$ Yi Deng, ${ }^{b}$ Xiao-hui Cao, ${ }^{a}$ Yue Wang ${ }^{a}$ and Ling Qin ${ }^{\mathrm{a}}$}

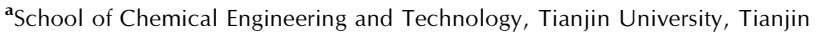
300072, People's Republic of China, and ${ }^{\mathbf{b}}$ College of Pharmaceuticals and Biotechnology, Tianjin University, Tianjin 300072, People's Republic of China

Correspondence e-mail: wwwwtju@yahoo.com.cn

Received 6 November 2007; accepted 5 December 2007

Key indicators: single-crystal X-ray study; $T=113 \mathrm{~K}$; mean $\sigma(\mathrm{C}-\mathrm{C})=0.003 \AA$; $R$ factor $=0.058 ; w R$ factor $=0.136 ;$ data-to-parameter ratio $=14.0$.

The title compound, $\mathrm{C}_{11} \mathrm{H}_{16} \mathrm{O}_{4}$, crystallizes with three molecules in the asymmetric unit. The cyclohexane ring adopts a chair conformation. Intermolecular $\mathrm{O}-\mathrm{H} \cdots \mathrm{O}$ hydrogen bonds are observed and these help to establish the crystal packing.

\section{Related literature}

For related literature, see: Stobbe (1893).

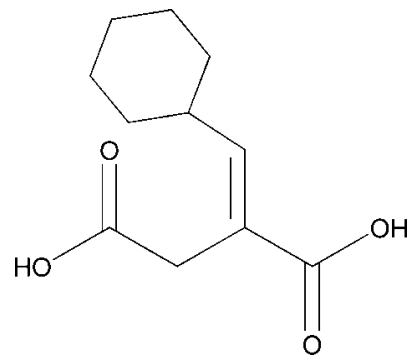

\section{Experimental}

Crystal data

$\mathrm{C}_{11} \mathrm{H}_{16} \mathrm{O}_{4}$

$M_{r}=212.24$

Monoclinic, $P 2_{1} / c$

$$
\begin{aligned}
& a=10.530(2) \AA \\
& b=18.762(4) \AA \\
& c=16.982(3) \AA
\end{aligned}
$$

$\beta=91.42(3)^{\circ}$

$V=3354.2(12) \AA^{3}$

$\mu=0.10 \mathrm{~mm}^{-1}$

$T=113(2) \mathrm{K}$

$Z=12$

Mo $K \alpha$ radiation

$0.10 \times 0.08 \times 0.06 \mathrm{~mm}$

Data collection

Rigaku Saturn diffractometer

Absorption correction: multi-scan (CrystalClear; Rigaku/MSC, 2005)

$T_{\min }=0.981, T_{\max }=0.994$

20420 measured reflections 5924 independent reflections 4780 reflections with $I>2 \sigma(I)$ $R_{\text {int }}=0.063$

Refinement

$R\left[F^{2}>2 \sigma\left(F^{2}\right)\right]=0.058$

$w R\left(F^{2}\right)=0.136$

$S=1.11$

5924 reflections

424 parameters

6 restraints

$\mathrm{H}$ atoms treated by a mixture of independent and constrained refinement

$\Delta \rho_{\max }=0.21{\mathrm{e} \AA^{-3}}^{-3}$

$\Delta \rho_{\min }=-0.27$ e $\AA^{-3}$

Table 1

Hydrogen-bond geometry $\left(\AA{ }^{\circ}\right)$.

\begin{tabular}{lllll}
\hline$D-\mathrm{H} \cdots A$ & $D-\mathrm{H}$ & $\mathrm{H} \cdots A$ & $D \cdots A$ & $D-\mathrm{H} \cdots A$ \\
\hline $\mathrm{O} 12-\mathrm{H} 12 \cdots \mathrm{O} 11^{\mathrm{i}}$ & $0.87(2)$ & $1.78(2)$ & $2.656(2)$ & $175(3)$ \\
$\mathrm{O} 10-\mathrm{H} 10 \cdots 9^{\mathrm{ii}}$ & $0.95(2)$ & $1.63(2)$ & $2.586(2)$ & $175(3)$ \\
$\mathrm{O} 8-\mathrm{H} 8 \cdots \mathrm{O} 4^{\mathrm{iii}}$ & $0.95(3)$ & $1.70(3)$ & $2.642(2)$ & $172(3)$ \\
$\mathrm{O} 5-\mathrm{H} 5 \cdots \mathrm{O} 2$ & $0.92(2)$ & $1.68(2)$ & $2.594(2)$ & $175(3)$ \\
$\mathrm{O} 3-\mathrm{H} 3 \cdots \mathrm{O} 7^{\text {iv }}$ & $0.90(2)$ & $1.75(2)$ & $2.644(2)$ & $173(3)$ \\
$\mathrm{O} 1-\mathrm{H} 1 \cdots \mathrm{O} 6$ & $0.93(2)$ & $1.66(2)$ & $2.591(2)$ & $175(3)$ \\
\hline
\end{tabular}

Symmetry codes: (i) $-x+1,-y+1,-z+2$; (ii) $-x,-y+1,-z+2$; (iii) $x-1, y, z$; (iv) $x+1, y, z$.

Data collection: CrystalClear (Rigaku/MSC, 2005); cell refinement: CrystalClear; data reduction: CrystalClear; program(s) used to solve structure: SHELXS97 (Sheldrick, 1997); program(s) used to refine structure: SHELXL97 (Sheldrick, 1997); molecular graphics: SHELXTL (Bruker, 1997); software used to prepare material for publication: SHELXTL.

Supplementary data and figures for this paper are available from the IUCr electronic archives (Reference: GW2033).

\title{
References
}

Bruker (1997). SHELXTL. Bruker AXS Inc., Madison, Wisconsin, USA.

Rigaku/MSC (2005). CrystalClear. Rigaku/MSC, The Woodlands, Texas, USA.

Sheldrick, G. M. (1997). SHELXS97 and SHELXL97. University of Göttingen, Germany.

Stobbe, H. (1893). Ber. Dtsch. Chem. Ges. 26, 2312. 


\section{supporting information}

Acta Cryst. (2008). E64, o336 [https://doi.org/10.1107/S1600536807065695]

\section{(E)-2-(Cyclohexylmethylene)succinic acid}

\section{Wei Wang, Yi Deng, Xiao-hui Cao, Yue Wang and Ling Qin}

\section{S1. Comment}

The title compound is obtained by the condensation between dimethylsuccinate and cyclohexanecarbaldehyde. We report here the crystal structure of the title compound (Fig. 1).

In the title compound, $\mathrm{C}_{11} \mathrm{H}_{16} \mathrm{O}_{4}$, the cyclohexane ring adopts chair conformation. Intermolecular $\mathrm{O}-\mathrm{H} \cdots \mathrm{O}$ hydrogen bonds are observed. They help to establish the crystal packing.

\section{S2. Experimental}

The title compound was prepared according to the method of Stobbe condensation (Stobbe et al.,1893). To a room temperature stirred solution of $50 \% \mathrm{NaH}(1.06 \mathrm{~g}, 0.022 \mathrm{~mol})$ in anhydrous toluene $(20 \mathrm{ml})$, a solution of dimethylsuccinate $(3.2 \mathrm{~g}, 0.022 \mathrm{~mol})$ and anhydrous methanol $(1 \mathrm{ml})$ in anhydrous toluene $(10 \mathrm{ml})$ was added dropwise. The mixture was stirred for $1 \mathrm{~h}$, and then a solution of cyclohexanecarbaldehyde $(2.24 \mathrm{~g}, 0.02 \mathrm{~mol})$ in anhydrous toluene (20 $\mathrm{ml}$ ) was added dropwise. The mixture was stirred at room temperature for $3 \mathrm{~h}$ and water $(30 \mathrm{ml})$ was added. The water phase was washed with ethyl acetatethree times and then sodium hydroxide ( $3 \mathrm{~g}, 0.075 \mathrm{~mol})$ was added. The mixture was refluxed for 4 and then acidified with hydrochloric acid to PH 2 and extracted with ethyl acetate. The organic layer was dried with anhydrous $\mathrm{Mg}_{2} \mathrm{SO}_{4}$, and then concentrated in vacuo. The title compound (3.65 g) was obtained in powder form in a yield of $86.1 \%$. Crystals of (I) were obtained by slow evaporation of a solution of ethanol (m.p. 477-478 K).

\section{S3. Refinement}

All $\mathrm{H}$ atoms were positioned geometrically $(\mathrm{C}-\mathrm{H}=0.95-0.99 \AA)$, and refined as riding with $U_{\text {iso }}(\mathrm{H})=1.2 U_{\text {eq }}($ carrier $)$ or $1.5_{\text {eq }}$ (methyl groups). 


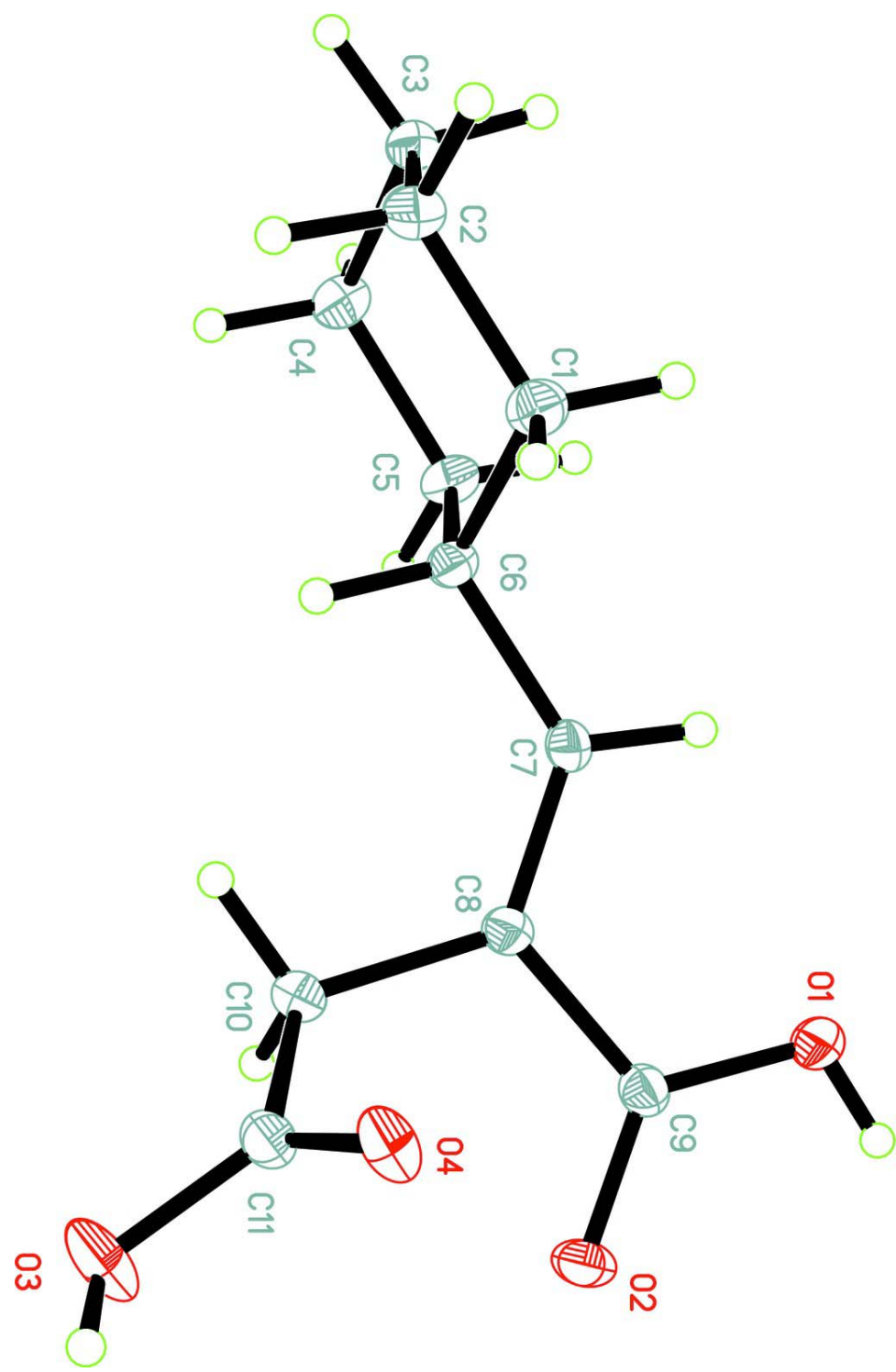

Figure 1

A view of the molecular of (I). Displacement ellopsoids are drawn at the $30 \%$ probability level and $\mathrm{H}$ atoms are shown as small spheres of arbitrary radii.

\section{(E)-2-(Cyclohexylmethylene)succinic acid}

\section{Crystal data}

$\mathrm{C}_{11} \mathrm{H}_{16} \mathrm{O}_{4}$

$M_{r}=212.24$

Monoclinic, $P 2_{1} / c$

$a=10.530(2) \AA$

$b=18.762(4) \AA$

$c=16.982(3) \AA$

$\beta=91.42(3)^{\circ}$

$V=3354.2(12) \AA^{3}$

$Z=12$

$F(000)=1368$
$D_{\mathrm{x}}=1.261 \mathrm{Mg} \mathrm{m}^{-3}$

Melting point $=477-478 \mathrm{~K}$

Mo $K \alpha$ radiation, $\lambda=0.71073 \AA$

Cell parameters from 266 reflections

$\theta=1.6-27.9^{\circ}$

$\mu=0.10 \mathrm{~mm}^{-1}$

$T=113 \mathrm{~K}$

Block, colorless

$0.10 \times 0.08 \times 0.06 \mathrm{~mm}$ 


\section{Data collection}

Rigaku Saturn diffractometer

Radiation source: fine-focus sealed tube Graphite monochromator $\omega$ scans

Absorption correction: multi-scan (CrystalClear; Rigaku/MSC, 2005)

$T_{\min }=0.981, T_{\max }=0.994$

\section{Refinement}

Refinement on $F^{2}$

Least-squares matrix: full

$R\left[F^{2}>2 \sigma\left(F^{2}\right)\right]=0.058$

$w R\left(F^{2}\right)=0.136$

$S=1.11$

5924 reflections

424 parameters

6 restraints

Primary atom site location: structure-invariant direct methods
20420 measured reflections

5924 independent reflections

4780 reflections with $I>2 \sigma(I)$

$R_{\text {int }}=0.063$

$\theta_{\max }=25.0^{\circ}, \theta_{\min }=1.6^{\circ}$

$h=-12 \rightarrow 12$

$k=-22 \rightarrow 17$

$l=-20 \rightarrow 20$

Secondary atom site location: difference Fourier map

Hydrogen site location: inferred from neighbouring sites

$\mathrm{H}$ atoms treated by a mixture of independent and constrained refinement

$w=1 /\left[\sigma^{2}\left(F_{\mathrm{o}}^{2}\right)+(0.0559 P)^{2}+0.7047 P\right]$ where $P=\left(F_{\mathrm{o}}^{2}+2 F_{\mathrm{c}}^{2}\right) / 3$

$(\Delta / \sigma)_{\max }=0.001$

$\Delta \rho_{\max }=0.21 \mathrm{e}^{-3}$

$\Delta \rho_{\min }=-0.27 \mathrm{e}^{-3}$

\section{Special details}

Geometry. All e.s.d.'s (except the e.s.d. in the dihedral angle between two 1.s. planes) are estimated using the full covariance matrix. The cell e.s.d.'s are taken into account individually in the estimation of e.s.d.'s in distances, angles and torsion angles; correlations between e.s.d.'s in cell parameters are only used when they are defined by crystal symmetry. An approximate (isotropic) treatment of cell e.s.d.'s is used for estimating e.s.d.'s involving l.s. planes.

Refinement. Refinement of $F^{2}$ against ALL reflections. The weighted $R$-factor $w R$ and goodness of fit $S$ are based on $F^{2}$, conventional $R$-factors $R$ are based on $F$, with $F$ set to zero for negative $F^{2}$. The threshold expression of $F^{2}>\sigma\left(F^{2}\right)$ is used only for calculating $R$-factors (gt) etc. and is not relevant to the choice of reflections for refinement. $R$-factors based on $F^{2}$ are statistically about twice as large as those based on $F$, and $R$ - factors based on ALL data will be even larger.

Fractional atomic coordinates and isotropic or equivalent isotropic displacement parameters $\left(\AA^{2}\right)$

\begin{tabular}{lllll}
\hline & $x$ & $y$ & $z$ & $U_{\text {iso }} * / U_{\text {eq }}$ \\
\hline O1 & $0.52956(15)$ & $0.70841(9)$ & $0.41522(9)$ & $0.0304(4)$ \\
H1 & $0.484(2)$ & $0.6721(13)$ & $0.4393(15)$ & $0.046^{*}$ \\
O2 & $0.66462(15)$ & $0.69150(9)$ & $0.51719(9)$ & $0.0332(4)$ \\
O3 & $0.99241(18)$ & $0.73367(10)$ & $0.54599(10)$ & $0.0411(5)$ \\
H3 & $1.041(3)$ & $0.6941(14)$ & $0.5465(18)$ & $0.062^{*}$ \\
O4 & $0.92339(15)$ & $0.68425(9)$ & $0.43354(9)$ & $0.0316(4)$ \\
O5 & $0.52767(15)$ & $0.59208(9)$ & $0.57875(9)$ & $0.0316(4)$ \\
H5 & $0.572(2)$ & $0.6279(13)$ & $0.5551(15)$ & $0.047^{*}$ \\
O6 & $0.39596(15)$ & $0.60645(9)$ & $0.47430(9)$ & $0.0302(4)$ \\
O7 & $0.13511(15)$ & $0.61815(9)$ & $0.55937(9)$ & $0.0329(4)$ \\
O8 & $0.06732(15)$ & $0.56926(10)$ & $0.44624(9)$ & $0.0298(4)$ \\
H8 & $0.022(3)$ & $0.6127(15)$ & $0.4416(15)$ & $0.045^{*}$ \\
O9 & $0.01165(15)$ & $0.54289(9)$ & $0.91554(9)$ & $0.0291(4)$ \\
O10 & $0.13307(16)$ & $0.55344(10)$ & $1.02487(9)$ & $0.0319(4)$ \\
H10 & $0.078(2)$ & $0.5177(13)$ & $1.0442(15)$ & $0.048^{*}$
\end{tabular}




\begin{tabular}{|c|c|c|c|c|}
\hline O11 & $0.38062(15)$ & $0.52336(9)$ & $0.94221(9)$ & $0.0314(4)$ \\
\hline $\mathrm{O} 12$ & $0.47862(16)$ & $0.59219(10)$ & $1.03214(10)$ & $0.0334(4)$ \\
\hline $\mathrm{H} 12$ & $0.529(3)$ & $0.5553(13)$ & $1.0388(17)$ & $0.050^{*}$ \\
\hline $\mathrm{C} 1$ & $0.7758(2)$ & $0.86243(13)$ & $0.23578(13)$ & $0.0286(6)$ \\
\hline $\mathrm{H} 1 \mathrm{~A}$ & 0.8336 & 0.8210 & 0.2322 & $0.034 *$ \\
\hline H1B & 0.6963 & 0.8508 & 0.2063 & $0.034 *$ \\
\hline $\mathrm{C} 2$ & $0.8377(2)$ & $0.92728(13)$ & $0.19892(13)$ & $0.0303(6)$ \\
\hline $\mathrm{H} 2 \mathrm{~A}$ & 0.9206 & 0.9365 & 0.2258 & $0.036^{*}$ \\
\hline $\mathrm{H} 2 \mathrm{~B}$ & 0.8536 & 0.9174 & 0.1428 & $0.036^{*}$ \\
\hline $\mathrm{C} 3$ & $0.7543(2)$ & $0.99311(13)$ & $0.20515(13)$ & $0.0283(6)$ \\
\hline $\mathrm{H} 3 \mathrm{~A}$ & 0.6752 & 0.9860 & 0.1734 & $0.034 *$ \\
\hline H3B & 0.7994 & 1.0348 & 0.1836 & $0.034 *$ \\
\hline $\mathrm{C} 4$ & $0.7209(2)$ & $1.00790(13)$ & $0.29086(13)$ & $0.0300(6)$ \\
\hline $\mathrm{H} 4 \mathrm{~A}$ & 0.7989 & 1.0211 & 0.3212 & $0.036^{*}$ \\
\hline $\mathrm{H} 4 \mathrm{~B}$ & 0.6611 & 1.0485 & 0.2928 & $0.036^{*}$ \\
\hline $\mathrm{C} 5$ & $0.6608(2)$ & $0.94265(12)$ & $0.32821(13)$ & $0.0290(6)$ \\
\hline $\mathrm{H} 5 \mathrm{~A}$ & 0.5780 & 0.9328 & 0.3015 & $0.035^{*}$ \\
\hline $\mathrm{H} 5 \mathrm{~B}$ & 0.6448 & 0.9527 & 0.3843 & $0.035^{*}$ \\
\hline $\mathrm{C} 6$ & $0.7462(2)$ & $0.87649(12)$ & $0.32232(12)$ & $0.0232(5)$ \\
\hline H6 & 0.8277 & 0.8859 & 0.3520 & $0.028^{*}$ \\
\hline $\mathrm{C} 7$ & $0.6815(2)$ & $0.81489(12)$ & $0.35909(12)$ & $0.0224(5)$ \\
\hline $\mathrm{H} 7$ & 0.6067 & 0.7984 & 0.3325 & $0.027^{*}$ \\
\hline $\mathrm{C} 8$ & $0.7153(2)$ & $0.77971(12)$ & $0.42489(12)$ & $0.0217(5)$ \\
\hline C9 & $0.6326(2)$ & $0.72300(12)$ & $0.45483(12)$ & $0.0233(5)$ \\
\hline $\mathrm{C} 10$ & $0.8316(2)$ & $0.79437(12)$ & $0.47521(13)$ & $0.0256(5)$ \\
\hline $\mathrm{H} 10 \mathrm{~A}$ & 0.8785 & 0.8348 & 0.4523 & $0.031^{*}$ \\
\hline $\mathrm{H} 10 \mathrm{~B}$ & 0.8050 & 0.8088 & 0.5284 & $0.031^{*}$ \\
\hline $\mathrm{C} 11$ & $0.9193(2)$ & $0.73159(13)$ & $0.48279(13)$ & $0.0258(5)$ \\
\hline $\mathrm{C} 12$ & $0.1708(2)$ & $0.47583(12)$ & $0.72032(13)$ & $0.0254(5)$ \\
\hline $\mathrm{H} 12 \mathrm{~A}$ & 0.2003 & 0.5118 & 0.7593 & $0.030^{*}$ \\
\hline $\mathrm{H} 12 \mathrm{~B}$ & 0.1197 & 0.5006 & 0.6790 & $0.030^{*}$ \\
\hline $\mathrm{C} 13$ & $0.0883(2)$ & $0.42064(14)$ & $0.76085(14)$ & $0.0316(6)$ \\
\hline $\mathrm{H} 13 \mathrm{~A}$ & 0.0163 & 0.4450 & 0.7859 & $0.038^{*}$ \\
\hline H13B & 0.0531 & 0.3871 & 0.7210 & $0.038^{*}$ \\
\hline $\mathrm{C} 14$ & $0.1639(2)$ & $0.37941(14)$ & $0.82281(13)$ & $0.0319(6)$ \\
\hline H14A & 0.1094 & 0.3418 & 0.8451 & $0.038^{*}$ \\
\hline H14B & 0.1899 & 0.4120 & 0.8661 & $0.038^{*}$ \\
\hline $\mathrm{C} 15$ & $0.2816(2)$ & $0.34522(13)$ & $0.78859(14)$ & $0.0342(6)$ \\
\hline $\mathrm{H} 15 \mathrm{~A}$ & 0.2554 & 0.3075 & 0.7508 & $0.041^{*}$ \\
\hline H15B & 0.3327 & 0.3227 & 0.8315 & $0.041^{*}$ \\
\hline $\mathrm{C} 16$ & $0.3624(2)$ & $0.40022(13)$ & $0.74674(14)$ & $0.0300(6)$ \\
\hline H16A & 0.4351 & 0.3759 & 0.7223 & $0.036^{*}$ \\
\hline H16B & 0.3969 & 0.4348 & 0.7858 & $0.036^{*}$ \\
\hline $\mathrm{C} 17$ & $0.2857(2)$ & $0.44026(12)$ & $0.68315(12)$ & $0.0221(5)$ \\
\hline H17 & 0.2536 & 0.4047 & 0.6436 & $0.026^{*}$ \\
\hline $\mathrm{C} 18$ & $0.3650(2)$ & $0.49360(12)$ & $0.64108(12)$ & $0.0210(5)$ \\
\hline $\mathrm{H} 18$ & 0.4416 & 0.5081 & 0.6670 & $0.025^{*}$ \\
\hline C19 & $0.3392(2)$ & $0.52292(12)$ & $0.57084(12)$ & $0.0220(5)$ \\
\hline
\end{tabular}




$\begin{array}{lllll}\mathrm{C} 20 & 0.4251(2) & 0.57730(12) & 0.53837(12) & 0.0218(5) \\ \mathrm{C} 21 & 0.2249(2) & 0.50708(12) & 0.51964(13) & 0.0241(5) \\ \mathrm{H} 21 \mathrm{~A} & 0.1769 & 0.4672 & 0.5429 & 0.029^{*} \\ \mathrm{H} 21 \mathrm{~B} & 0.2529 & 0.4918 & 0.4670 & 0.029^{*} \\ \mathrm{C} 22 & 0.1394(2) & 0.57048(13) & 0.51064(12) & 0.0245(5) \\ \mathrm{C} 23 & 0.1341(2) & 0.74770(13) & 0.74597(15) & 0.0337(6) \\ \mathrm{H} 23 \mathrm{~A} & 0.0935 & 0.7147 & 0.7074 & 0.040^{*} \\ \mathrm{H} 23 \mathrm{~B} & 0.0662 & 0.7703 & 0.7766 & 0.040^{*} \\ \mathrm{C} 24 & 0.2075(3) & 0.80494(14) & 0.70232(16) & 0.0406(7) \\ \mathrm{H} 24 \mathrm{~A} & 0.1494 & 0.8293 & 0.6643 & 0.049^{*} \\ \mathrm{H} 24 \mathrm{~B} & 0.2396 & 0.8409 & 0.7405 & 0.049^{*} \\ \mathrm{C} 25 & 0.3181(2) & 0.77368(15) & 0.65866(14) & 0.0392(7) \\ \mathrm{H} 25 \mathrm{~A} & 0.2850 & 0.7438 & 0.6147 & 0.047^{*} \\ \mathrm{H} 25 \mathrm{~B} & 0.3683 & 0.8129 & 0.6359 & 0.047^{*} \\ \mathrm{C} 26 & 0.4034(2) & 0.72914(14) & 0.71173(14) & 0.0330(6) \\ \mathrm{H} 26 \mathrm{~A} & 0.4466 & 0.7604 & 0.7510 & 0.040^{*} \\ \mathrm{H} 26 \mathrm{~B} & 0.4695 & 0.7062 & 0.6799 & 0.040^{*} \\ \mathrm{C} 27 & 0.3288(2) & 0.67178(13) & 0.75447(14) & 0.0279(6) \\ \mathrm{H} 27 \mathrm{~A} & 0.3868 & 0.6449 & 0.7904 & 0.033^{*} \\ \mathrm{H} 27 \mathrm{~B} & 0.2917 & 0.6379 & 0.7156 & 0.033^{*} \\ \mathrm{C} 28 & 0.2228(2) & 0.70562(12) & 0.80172(12) & 0.0232(5) \\ \mathrm{H} 28 & 0.2624 & 0.7398 & 0.8403 & 0.028^{*} \\ \mathrm{C} 29 & 0.1525(2) & 0.65043(12) & 0.84662(12) & 0.0227(5) \\ \mathrm{H} 29 & 0.0754 & 0.6334 & 0.8233 & 0.027^{*} \\ \mathrm{C} 30 & 0.1871(2) & 0.62253(12) & 0.91624(12) & 0.0222(5) \\ \mathrm{C} 31 & 0.1035(2) & 0.56922(12) & 0.95195(12) & 0.0214(5) \\ \mathrm{C} 32 & 0.3049(2) & 0.64220(13) & 0.96253(13) & 0.0258(5) \\ \mathrm{H} 32 \mathrm{~A} & 0.2806 & 0.6630 & 1.0136 & 0.031^{*} \\ \mathrm{H} 32 \mathrm{~B} & 0.3520 & 0.6790 & 0.9333 & 0.031^{*} \\ \mathrm{C} 33 & 0.3907(2) & 0.57935(13) & 0.97767(12) & 0.0247(5)\end{array}$

Atomic displacement parameters $\left(A^{2}\right)$

\begin{tabular}{lllllll}
\hline & $U^{11}$ & $U^{22}$ & $U^{33}$ & $U^{12}$ & $U^{13}$ & $U^{23}$ \\
\hline O1 & $0.0250(9)$ & $0.0345(11)$ & $0.0316(9)$ & $-0.0096(8)$ & $-0.0043(8)$ & $0.0093(8)$ \\
O2 & $0.0306(10)$ & $0.0391(11)$ & $0.0297(8)$ & $-0.0028(8)$ & $-0.0050(8)$ & $0.0159(8)$ \\
O3 & $0.0440(12)$ & $0.0446(12)$ & $0.0337(9)$ & $0.0166(9)$ & $-0.0211(9)$ & $-0.0119(9)$ \\
O4 & $0.0300(10)$ & $0.0369(11)$ & $0.0275(8)$ & $0.0057(8)$ & $-0.0083(8)$ & $-0.0069(8)$ \\
O5 & $0.0248(10)$ & $0.0374(11)$ & $0.0323(9)$ & $-0.0098(8)$ & $-0.0041(8)$ & $0.0096(8)$ \\
O6 & $0.0281(9)$ & $0.0362(10)$ & $0.0262(8)$ & $-0.0030(8)$ & $-0.0017(7)$ & $0.0101(7)$ \\
O7 & $0.0321(10)$ & $0.0372(11)$ & $0.0288(8)$ & $0.0094(8)$ & $-0.0097(8)$ & $-0.0087(8)$ \\
O8 & $0.0272(9)$ & $0.0369(11)$ & $0.0246(8)$ & $0.0041(8)$ & $-0.0087(7)$ & $-0.0017(7)$ \\
O9 & $0.0263(9)$ & $0.0322(10)$ & $0.0286(8)$ & $-0.0066(8)$ & $-0.0060(8)$ & $0.0064(7)$ \\
O10 & $0.0312(10)$ & $0.0412(11)$ & $0.0231(8)$ & $-0.0089(8)$ & $-0.0023(7)$ & $0.0107(7)$ \\
O11 & $0.0306(10)$ & $0.0321(10)$ & $0.0312(8)$ & $0.0027(8)$ & $-0.0079(8)$ & $-0.0075(8)$ \\
O12 & $0.0295(10)$ & $0.0379(11)$ & $0.0321(9)$ & $0.0044(8)$ & $-0.0141(8)$ & $-0.0088(8)$ \\
C1 & $0.0292(13)$ & $0.0280(14)$ & $0.0286(12)$ & $-0.0017(11)$ & $0.0026(11)$ & $0.0027(10)$ \\
C2 & $0.0317(14)$ & $0.0348(15)$ & $0.0245(11)$ & $-0.0047(11)$ & $0.0027(11)$ & $0.0037(10)$
\end{tabular}




\begin{tabular}{|c|c|c|c|c|c|c|}
\hline $\mathrm{C} 3$ & $0.0277(13)$ & $0.0287(14)$ & $0.0284(12)$ & $-0.0051(10)$ & $-0.0045(11)$ & $0.0075(10)$ \\
\hline $\mathrm{C} 4$ & $0.0319(14)$ & $0.0266(14)$ & $0.0316(12)$ & $-0.0028(11)$ & $0.0041(11)$ & $0.0023(10)$ \\
\hline $\mathrm{C} 5$ & $0.0299(13)$ & $0.0279(14)$ & $0.0296(12)$ & $-0.0028(11)$ & $0.0057(11)$ & $0.0050(10)$ \\
\hline C6 & $0.0201(12)$ & $0.0248(13)$ & $0.0246(11)$ & $-0.0032(10)$ & $-0.0011(10)$ & $0.0063(10)$ \\
\hline $\mathrm{C} 7$ & $0.0181(12)$ & $0.0259(13)$ & $0.0231(11)$ & $-0.0026(10)$ & $-0.0025(10)$ & $0.0020(10)$ \\
\hline $\mathrm{C} 8$ & $0.0212(12)$ & $0.0224(12)$ & $0.0215(10)$ & $0.0004(9)$ & $-0.0011(10)$ & $0.0026(9)$ \\
\hline C9 & $0.0213(13)$ & $0.0263(13)$ & $0.0222(11)$ & $-0.0002(10)$ & $-0.0013(10)$ & $0.0009(10)$ \\
\hline $\mathrm{C} 10$ & $0.0283(13)$ & $0.0255(14)$ & $0.0228(11)$ & $-0.0017(10)$ & $-0.0040(10)$ & $0.0032(10)$ \\
\hline $\mathrm{C} 11$ & $0.0229(13)$ & $0.0318(14)$ & $0.0225(11)$ & $-0.0025(10)$ & $-0.0019(10)$ & $0.0027(10)$ \\
\hline $\mathrm{C} 12$ & $0.0252(13)$ & $0.0252(13)$ & $0.0258(11)$ & $0.0033(10)$ & $0.0013(10)$ & $0.0053(10)$ \\
\hline $\mathrm{C} 13$ & $0.0259(13)$ & $0.0401(16)$ & $0.0292(12)$ & $-0.0020(11)$ & $0.0049(11)$ & $0.0071(11)$ \\
\hline $\mathrm{C} 14$ & $0.0338(14)$ & $0.0357(15)$ & $0.0262(12)$ & $-0.0073(11)$ & $0.0027(11)$ & $0.0068(11)$ \\
\hline $\mathrm{C} 15$ & $0.0406(15)$ & $0.0293(14)$ & $0.0325(13)$ & $0.0038(12)$ & $-0.0004(12)$ & $0.0100(11)$ \\
\hline $\mathrm{C} 16$ & $0.0286(14)$ & $0.0307(14)$ & $0.0307(12)$ & $0.0042(11)$ & $0.0028(11)$ & $0.0063(11)$ \\
\hline $\mathrm{C} 17$ & $0.0244(12)$ & $0.0190(12)$ & $0.0228(10)$ & $-0.0013(9)$ & $0.0009(10)$ & $-0.0001(9)$ \\
\hline $\mathrm{C} 18$ & $0.0171(11)$ & $0.0229(13)$ & $0.0231(11)$ & $0.0008(9)$ & $-0.0010(9)$ & $-0.0024(9)$ \\
\hline C19 & $0.0204(12)$ & $0.0216(12)$ & $0.0242(11)$ & $0.0035(9)$ & $0.0021(10)$ & $0.0005(9)$ \\
\hline $\mathrm{C} 20$ & $0.0176(12)$ & $0.0255(13)$ & $0.0223(11)$ & $0.0025(9)$ & $-0.0010(10)$ & $-0.0001(9)$ \\
\hline $\mathrm{C} 21$ & $0.0237(12)$ & $0.0253(13)$ & $0.0232(11)$ & $-0.0025(10)$ & $0.0012(10)$ & $0.0009(9)$ \\
\hline $\mathrm{C} 22$ & $0.0203(12)$ & $0.0314(14)$ & $0.0218(11)$ & $-0.0025(10)$ & $0.0017(10)$ & $0.0029(10)$ \\
\hline $\mathrm{C} 23$ & $0.0331(14)$ & $0.0282(14)$ & $0.0398(14)$ & $0.0045(11)$ & $0.0011(12)$ & $0.0126(11)$ \\
\hline $\mathrm{C} 24$ & $0.0395(16)$ & $0.0344(16)$ & $0.0474(15)$ & $-0.0022(13)$ & $-0.0086(13)$ & $0.0212(13)$ \\
\hline $\mathrm{C} 25$ & $0.0419(16)$ & $0.0454(17)$ & $0.0299(13)$ & $-0.0208(13)$ & $-0.0069(12)$ & $0.0131(12)$ \\
\hline $\mathrm{C} 26$ & $0.0290(14)$ & $0.0380(16)$ & $0.0321(13)$ & $-0.0052(11)$ & $0.0036(12)$ & $0.0040(11)$ \\
\hline $\mathrm{C} 27$ & $0.0245(13)$ & $0.0267(14)$ & $0.0325(12)$ & $0.0002(10)$ & $0.0005(11)$ & $0.0031(10)$ \\
\hline $\mathrm{C} 28$ & $0.0277(13)$ & $0.0222(13)$ & $0.0197(10)$ & $-0.0035(10)$ & $-0.0023(10)$ & $0.0008(9)$ \\
\hline $\mathrm{C} 29$ & $0.0236(12)$ & $0.0221(13)$ & $0.0223(11)$ & $-0.0002(10)$ & $-0.0001(10)$ & $-0.0022(9)$ \\
\hline $\mathrm{C} 30$ & $0.0236(12)$ & $0.0213(12)$ & $0.0217(10)$ & $0.0019(10)$ & $0.0013(10)$ & $0.0003(9)$ \\
\hline C31 & $0.0199(12)$ & $0.0252(13)$ & $0.0190(10)$ & $0.0026(10)$ & $-0.0008(10)$ & $0.0019(9)$ \\
\hline $\mathrm{C} 32$ & $0.0286(13)$ & $0.0275(14)$ & $0.0213(11)$ & $-0.0037(10)$ & $-0.0022(10)$ & $0.0013(10)$ \\
\hline C33 & $0.0201(12)$ & $0.0345(15)$ & $0.0196(10)$ & $-0.0031(10)$ & $0.0017(10)$ & $0.0015(10)$ \\
\hline
\end{tabular}

Geometric parameters $\left(\AA,{ }^{\circ}\right)$

\begin{tabular}{llll}
\hline $\mathrm{O} 1-\mathrm{C} 9$ & $1.292(3)$ & $\mathrm{C} 13-\mathrm{C} 14$ & $1.516(3)$ \\
$\mathrm{O} 1-\mathrm{H} 1$ & $0.93(2)$ & $\mathrm{C} 13-\mathrm{H} 13 \mathrm{~A}$ & 0.9900 \\
$\mathrm{O} 2-\mathrm{C} 9$ & $1.252(3)$ & $\mathrm{C} 13-\mathrm{H} 13 \mathrm{~B}$ & 0.9900 \\
$\mathrm{O} 3-\mathrm{C} 11$ & $1.306(3)$ & $\mathrm{C} 14-\mathrm{C} 15$ & $1.524(3)$ \\
$\mathrm{O} 3-\mathrm{H} 3$ & $0.90(2)$ & $\mathrm{C} 14-\mathrm{H} 14 \mathrm{~A}$ & 0.9900 \\
$\mathrm{O} 4-\mathrm{C} 11$ & $1.222(3)$ & $\mathrm{C} 14-\mathrm{H} 14 \mathrm{~B}$ & 0.9900 \\
$\mathrm{O} 5-\mathrm{C} 20$ & $1.295(3)$ & $\mathrm{C} 15-\mathrm{C} 16$ & $1.525(3)$ \\
$\mathrm{O}-\mathrm{H} 5$ & $0.92(2)$ & $\mathrm{C} 15-\mathrm{H} 15 \mathrm{~A}$ & 0.9900 \\
$\mathrm{O} 6-\mathrm{C} 20$ & $1.249(3)$ & $\mathrm{C} 15-\mathrm{H} 15 \mathrm{~B}$ & 0.9900 \\
$\mathrm{O} 7-\mathrm{C} 22$ & $1.220(3)$ & $\mathrm{C} 16-\mathrm{C} 17$ & $1.530(3)$ \\
$\mathrm{O} 8-\mathrm{C} 22$ & $1.316(3)$ & $\mathrm{C} 16-\mathrm{H} 16 \mathrm{~A}$ & 0.9900 \\
$\mathrm{O} 8-\mathrm{H} 8$ & $0.95(3)$ & $\mathrm{C} 16-\mathrm{H} 16 \mathrm{~B}$ & 0.9900 \\
$\mathrm{O} 9-\mathrm{C} 31$ & $1.238(3)$ & $\mathrm{C} 17-\mathrm{C} 18$ & $1.496(3)$ \\
$\mathrm{O} 10-\mathrm{C} 31$ & $1.303(3)$ & $\mathrm{C} 17-\mathrm{H} 17$ & 1.0000
\end{tabular}




\begin{tabular}{|c|c|c|c|}
\hline $\mathrm{O} 10-\mathrm{H} 10$ & $0.95(2)$ & $\mathrm{C} 18-\mathrm{C} 19$ & $1.335(3)$ \\
\hline $\mathrm{O} 11-\mathrm{C} 33$ & $1.214(3)$ & $\mathrm{C} 18-\mathrm{H} 18$ & 0.9500 \\
\hline $\mathrm{O} 12-\mathrm{C} 33$ & $1.315(3)$ & $\mathrm{C} 19-\mathrm{C} 20$ & $1.479(3)$ \\
\hline $\mathrm{O} 12-\mathrm{H} 12$ & $0.87(2)$ & $\mathrm{C} 19-\mathrm{C} 21$ & $1.497(3)$ \\
\hline $\mathrm{C} 1-\mathrm{C} 2$ & $1.522(3)$ & $\mathrm{C} 21-\mathrm{C} 22$ & $1.498(3)$ \\
\hline $\mathrm{C} 1-\mathrm{C} 6$ & $1.533(3)$ & $\mathrm{C} 21-\mathrm{H} 21 \mathrm{~A}$ & 0.9900 \\
\hline $\mathrm{C} 1-\mathrm{H} 1 \mathrm{~A}$ & 0.9900 & $\mathrm{C} 21-\mathrm{H} 21 \mathrm{~B}$ & 0.9900 \\
\hline $\mathrm{C} 1-\mathrm{H} 1 \mathrm{~B}$ & 0.9900 & $\mathrm{C} 23-\mathrm{C} 24$ & $1.526(3)$ \\
\hline $\mathrm{C} 2-\mathrm{C} 3$ & $1.520(3)$ & $\mathrm{C} 23-\mathrm{C} 28$ & $1.533(3)$ \\
\hline $\mathrm{C} 2-\mathrm{H} 2 \mathrm{~A}$ & 0.9900 & $\mathrm{C} 23-\mathrm{H} 23 \mathrm{~A}$ & 0.9900 \\
\hline $\mathrm{C} 2-\mathrm{H} 2 \mathrm{~B}$ & 0.9900 & $\mathrm{C} 23-\mathrm{H} 23 \mathrm{~B}$ & 0.9900 \\
\hline $\mathrm{C} 3-\mathrm{C} 4$ & $1.531(3)$ & $\mathrm{C} 24-\mathrm{C} 25$ & $1.514(4)$ \\
\hline $\mathrm{C} 3-\mathrm{H} 3 \mathrm{~A}$ & 0.9900 & $\mathrm{C} 24-\mathrm{H} 24 \mathrm{~A}$ & 0.9900 \\
\hline $\mathrm{C} 3-\mathrm{H} 3 \mathrm{~B}$ & 0.9900 & $\mathrm{C} 24-\mathrm{H} 24 \mathrm{~B}$ & 0.9900 \\
\hline $\mathrm{C} 4-\mathrm{C} 5$ & $1.524(3)$ & $\mathrm{C} 25-\mathrm{C} 26$ & $1.509(4)$ \\
\hline $\mathrm{C} 4-\mathrm{H} 4 \mathrm{~A}$ & 0.9900 & $\mathrm{C} 25-\mathrm{H} 25 \mathrm{~A}$ & 0.9900 \\
\hline $\mathrm{C} 4-\mathrm{H} 4 \mathrm{~B}$ & 0.9900 & $\mathrm{C} 25-\mathrm{H} 25 \mathrm{~B}$ & 0.9900 \\
\hline $\mathrm{C} 5-\mathrm{C} 6$ & $1.538(3)$ & $\mathrm{C} 26-\mathrm{C} 27$ & $1.526(3)$ \\
\hline $\mathrm{C} 5-\mathrm{H} 5 \mathrm{~A}$ & 0.9900 & $\mathrm{C} 26-\mathrm{H} 26 \mathrm{~A}$ & 0.9900 \\
\hline C5-H5B & 0.9900 & $\mathrm{C} 26-\mathrm{H} 26 \mathrm{~B}$ & 0.9900 \\
\hline $\mathrm{C} 6-\mathrm{C} 7$ & $1.487(3)$ & $\mathrm{C} 27-\mathrm{C} 28$ & $1.529(3)$ \\
\hline $\mathrm{C} 6-\mathrm{H} 6$ & 1.0000 & $\mathrm{C} 27-\mathrm{H} 27 \mathrm{~A}$ & 0.9900 \\
\hline $\mathrm{C} 7-\mathrm{C} 8$ & $1.338(3)$ & $\mathrm{C} 27-\mathrm{H} 27 \mathrm{~B}$ & 0.9900 \\
\hline $\mathrm{C} 7-\mathrm{H} 7$ & 0.9500 & $\mathrm{C} 28-\mathrm{C} 29$ & $1.493(3)$ \\
\hline $\mathrm{C} 8-\mathrm{C} 9$ & $1.474(3)$ & $\mathrm{C} 28-\mathrm{H} 28$ & 1.0000 \\
\hline $\mathrm{C} 8-\mathrm{C} 10$ & $1.502(3)$ & $\mathrm{C} 29-\mathrm{C} 30$ & $1.335(3)$ \\
\hline $\mathrm{C} 10-\mathrm{C} 11$ & $1.500(3)$ & $\mathrm{C} 29-\mathrm{H} 29$ & 0.9500 \\
\hline $\mathrm{C} 10-\mathrm{H} 10 \mathrm{~A}$ & 0.9900 & $\mathrm{C} 30-\mathrm{C} 31$ & $1.472(3)$ \\
\hline $\mathrm{C} 10-\mathrm{H} 10 \mathrm{~B}$ & 0.9900 & $\mathrm{C} 30-\mathrm{C} 32$ & $1.499(3)$ \\
\hline $\mathrm{C} 12-\mathrm{C} 13$ & $1.526(3)$ & $\mathrm{C} 32-\mathrm{C} 33$ & $1.504(3)$ \\
\hline $\mathrm{C} 12-\mathrm{C} 17$ & $1.532(3)$ & $\mathrm{C} 32-\mathrm{H} 32 \mathrm{~A}$ & 0.9900 \\
\hline $\mathrm{C} 12-\mathrm{H} 12 \mathrm{~A}$ & 0.9900 & $\mathrm{C} 32-\mathrm{H} 32 \mathrm{~B}$ & 0.9900 \\
\hline $\mathrm{C} 12-\mathrm{H} 12 \mathrm{~B}$ & 0.9900 & & \\
\hline $\mathrm{C} 9-\mathrm{O} 1-\mathrm{H} 1$ & $111.1(17)$ & $\mathrm{C} 15-\mathrm{C} 16-\mathrm{C} 17$ & $111.7(2)$ \\
\hline $\mathrm{C} 11-\mathrm{O} 3-\mathrm{H} 3$ & $108(2)$ & $\mathrm{C} 15-\mathrm{C} 16-\mathrm{H} 16 \mathrm{~A}$ & 109.3 \\
\hline $\mathrm{C} 20-\mathrm{O} 5-\mathrm{H} 5$ & $110.8(18)$ & $\mathrm{C} 17-\mathrm{C} 16-\mathrm{H} 16 \mathrm{~A}$ & 109.3 \\
\hline $\mathrm{C} 22-\mathrm{O} 8-\mathrm{H} 8$ & $109.4(17)$ & $\mathrm{C} 15-\mathrm{C} 16-\mathrm{H} 16 \mathrm{~B}$ & 109.3 \\
\hline $\mathrm{C} 31-\mathrm{O} 10-\mathrm{H} 10$ & $110.7(17)$ & $\mathrm{C} 17-\mathrm{C} 16-\mathrm{H} 16 \mathrm{~B}$ & 109.3 \\
\hline $\mathrm{C} 33-\mathrm{O} 12-\mathrm{H} 12$ & $111(2)$ & $\mathrm{H} 16 \mathrm{~A}-\mathrm{C} 16-\mathrm{H} 16 \mathrm{~B}$ & 107.9 \\
\hline $\mathrm{C} 2-\mathrm{C} 1-\mathrm{C} 6$ & $110.85(19)$ & $\mathrm{C} 18-\mathrm{C} 17-\mathrm{C} 16$ & $112.02(19)$ \\
\hline $\mathrm{C} 2-\mathrm{C} 1-\mathrm{H} 1 \mathrm{~A}$ & 109.5 & $\mathrm{C} 18-\mathrm{C} 17-\mathrm{C} 12$ & $111.17(18)$ \\
\hline $\mathrm{C} 6-\mathrm{C} 1-\mathrm{H} 1 \mathrm{~A}$ & 109.5 & $\mathrm{C} 16-\mathrm{C} 17-\mathrm{C} 12$ & $109.38(17)$ \\
\hline $\mathrm{C} 2-\mathrm{C} 1-\mathrm{H} 1 \mathrm{~B}$ & 109.5 & $\mathrm{C} 18-\mathrm{C} 17-\mathrm{H} 17$ & 108.0 \\
\hline $\mathrm{C} 6-\mathrm{C} 1-\mathrm{H} 1 \mathrm{~B}$ & 109.5 & $\mathrm{C} 16-\mathrm{C} 17-\mathrm{H} 17$ & 108.0 \\
\hline $\mathrm{H} 1 \mathrm{~A}-\mathrm{C} 1-\mathrm{H} 1 \mathrm{~B}$ & 108.1 & $\mathrm{C} 12-\mathrm{C} 17-\mathrm{H} 17$ & 108.0 \\
\hline $\mathrm{C} 3-\mathrm{C} 2-\mathrm{C} 1$ & $111.55(19)$ & $\mathrm{C} 19-\mathrm{C} 18-\mathrm{C} 17$ & $126.8(2)$ \\
\hline $\mathrm{C} 3-\mathrm{C} 2-\mathrm{H} 2 \mathrm{~A}$ & 109.3 & $\mathrm{C} 19-\mathrm{C} 18-\mathrm{H} 18$ & 116.6 \\
\hline
\end{tabular}




\begin{tabular}{|c|c|c|c|}
\hline $\mathrm{C} 1-\mathrm{C} 2-\mathrm{H} 2 \mathrm{~A}$ & 109.3 & $\mathrm{C} 17-\mathrm{C} 18-\mathrm{H} 18$ & 116.6 \\
\hline $\mathrm{C} 3-\mathrm{C} 2-\mathrm{H} 2 \mathrm{~B}$ & 109.3 & $\mathrm{C} 18-\mathrm{C} 19-\mathrm{C} 20$ & $120.3(2)$ \\
\hline $\mathrm{C} 1-\mathrm{C} 2-\mathrm{H} 2 \mathrm{~B}$ & 109.3 & $\mathrm{C} 18-\mathrm{C} 19-\mathrm{C} 21$ & $125.4(2)$ \\
\hline $\mathrm{H} 2 \mathrm{~A}-\mathrm{C} 2-\mathrm{H} 2 \mathrm{~B}$ & 108.0 & $\mathrm{C} 20-\mathrm{C} 19-\mathrm{C} 21$ & $114.26(19)$ \\
\hline $\mathrm{C} 2-\mathrm{C} 3-\mathrm{C} 4$ & $111.1(2)$ & $\mathrm{O} 6-\mathrm{C} 20-\mathrm{O} 5$ & $123.4(2)$ \\
\hline $\mathrm{C} 2-\mathrm{C} 3-\mathrm{H} 3 \mathrm{~A}$ & 109.4 & $\mathrm{O} 6-\mathrm{C} 20-\mathrm{C} 19$ & $119.2(2)$ \\
\hline $\mathrm{C} 4-\mathrm{C} 3-\mathrm{H} 3 \mathrm{~A}$ & 109.4 & $\mathrm{O} 5-\mathrm{C} 20-\mathrm{C} 19$ & $117.40(19)$ \\
\hline $\mathrm{C} 2-\mathrm{C} 3-\mathrm{H} 3 \mathrm{~B}$ & 109.4 & $\mathrm{C} 19-\mathrm{C} 21-\mathrm{C} 22$ & $111.99(19)$ \\
\hline $\mathrm{C} 4-\mathrm{C} 3-\mathrm{H} 3 \mathrm{~B}$ & 109.4 & $\mathrm{C} 19-\mathrm{C} 21-\mathrm{H} 21 \mathrm{~A}$ & 109.2 \\
\hline $\mathrm{H} 3 \mathrm{~A}-\mathrm{C} 3-\mathrm{H} 3 \mathrm{~B}$ & 108.0 & $\mathrm{C} 22-\mathrm{C} 21-\mathrm{H} 21 \mathrm{~A}$ & 109.2 \\
\hline $\mathrm{C} 5-\mathrm{C} 4-\mathrm{C} 3$ & $111.0(2)$ & $\mathrm{C} 19-\mathrm{C} 21-\mathrm{H} 21 \mathrm{~B}$ & 109.2 \\
\hline $\mathrm{C} 5-\mathrm{C} 4-\mathrm{H} 4 \mathrm{~A}$ & 109.4 & $\mathrm{C} 22-\mathrm{C} 21-\mathrm{H} 21 \mathrm{~B}$ & 109.2 \\
\hline $\mathrm{C} 3-\mathrm{C} 4-\mathrm{H} 4 \mathrm{~A}$ & 109.4 & $\mathrm{H} 21 \mathrm{~A}-\mathrm{C} 21-\mathrm{H} 21 \mathrm{~B}$ & 107.9 \\
\hline $\mathrm{C} 5-\mathrm{C} 4-\mathrm{H} 4 \mathrm{~B}$ & 109.4 & $\mathrm{O} 7-\mathrm{C} 22-\mathrm{O} 8$ & $123.1(2)$ \\
\hline $\mathrm{C} 3-\mathrm{C} 4-\mathrm{H} 4 \mathrm{~B}$ & 109.4 & $\mathrm{O} 7-\mathrm{C} 22-\mathrm{C} 21$ & $123.0(2)$ \\
\hline $\mathrm{H} 4 \mathrm{~A}-\mathrm{C} 4-\mathrm{H} 4 \mathrm{~B}$ & 108.0 & $\mathrm{O} 8-\mathrm{C} 22-\mathrm{C} 21$ & $113.8(2)$ \\
\hline $\mathrm{C} 4-\mathrm{C} 5-\mathrm{C} 6$ & $111.80(18)$ & $\mathrm{C} 24-\mathrm{C} 23-\mathrm{C} 28$ & $110.8(2)$ \\
\hline $\mathrm{C} 4-\mathrm{C} 5-\mathrm{H} 5 \mathrm{~A}$ & 109.3 & $\mathrm{C} 24-\mathrm{C} 23-\mathrm{H} 23 \mathrm{~A}$ & 109.5 \\
\hline $\mathrm{C} 6-\mathrm{C} 5-\mathrm{H} 5 \mathrm{~A}$ & 109.3 & $\mathrm{C} 28-\mathrm{C} 23-\mathrm{H} 23 \mathrm{~A}$ & 109.5 \\
\hline $\mathrm{C} 4-\mathrm{C} 5-\mathrm{H} 5 \mathrm{~B}$ & 109.3 & $\mathrm{C} 24-\mathrm{C} 23-\mathrm{H} 23 \mathrm{~B}$ & 109.5 \\
\hline $\mathrm{C} 6-\mathrm{C} 5-\mathrm{H} 5 \mathrm{~B}$ & 109.3 & $\mathrm{C} 28-\mathrm{C} 23-\mathrm{H} 23 \mathrm{~B}$ & 109.5 \\
\hline $\mathrm{H} 5 \mathrm{~A}-\mathrm{C} 5-\mathrm{H} 5 \mathrm{~B}$ & 107.9 & $\mathrm{H} 23 \mathrm{~A}-\mathrm{C} 23-\mathrm{H} 23 \mathrm{~B}$ & 108.1 \\
\hline $\mathrm{C} 7-\mathrm{C} 6-\mathrm{C} 1$ & $112.03(19)$ & $\mathrm{C} 25-\mathrm{C} 24-\mathrm{C} 23$ & $111.7(2)$ \\
\hline $\mathrm{C} 7-\mathrm{C} 6-\mathrm{C} 5$ & $108.97(17)$ & $\mathrm{C} 25-\mathrm{C} 24-\mathrm{H} 24 \mathrm{~A}$ & 109.3 \\
\hline $\mathrm{C} 1-\mathrm{C} 6-\mathrm{C} 5$ & $109.54(19)$ & $\mathrm{C} 23-\mathrm{C} 24-\mathrm{H} 24 \mathrm{~A}$ & 109.3 \\
\hline $\mathrm{C} 7-\mathrm{C} 6-\mathrm{H} 6$ & 108.7 & $\mathrm{C} 25-\mathrm{C} 24-\mathrm{H} 24 \mathrm{~B}$ & 109.3 \\
\hline $\mathrm{C} 1-\mathrm{C} 6-\mathrm{H} 6$ & 108.7 & $\mathrm{C} 23-\mathrm{C} 24-\mathrm{H} 24 \mathrm{~B}$ & 109.3 \\
\hline $\mathrm{C} 5-\mathrm{C} 6-\mathrm{H} 6$ & 108.7 & $\mathrm{H} 24 \mathrm{~A}-\mathrm{C} 24-\mathrm{H} 24 \mathrm{~B}$ & 107.9 \\
\hline $\mathrm{C} 8-\mathrm{C} 7-\mathrm{C} 6$ & $128.2(2)$ & $\mathrm{C} 26-\mathrm{C} 25-\mathrm{C} 24$ & $112.1(2)$ \\
\hline $\mathrm{C} 8-\mathrm{C} 7-\mathrm{H} 7$ & 115.9 & $\mathrm{C} 26-\mathrm{C} 25-\mathrm{H} 25 \mathrm{~A}$ & 109.2 \\
\hline $\mathrm{C} 6-\mathrm{C} 7-\mathrm{H} 7$ & 115.9 & $\mathrm{C} 24-\mathrm{C} 25-\mathrm{H} 25 \mathrm{~A}$ & 109.2 \\
\hline $\mathrm{C} 7-\mathrm{C} 8-\mathrm{C} 9$ & $119.8(2)$ & $\mathrm{C} 26-\mathrm{C} 25-\mathrm{H} 25 \mathrm{~B}$ & 109.2 \\
\hline $\mathrm{C} 7-\mathrm{C} 8-\mathrm{C} 10$ & $125.5(2)$ & $\mathrm{C} 24-\mathrm{C} 25-\mathrm{H} 25 \mathrm{~B}$ & 109.2 \\
\hline $\mathrm{C} 9-\mathrm{C} 8-\mathrm{C} 10$ & $114.64(19)$ & $\mathrm{H} 25 \mathrm{~A}-\mathrm{C} 25-\mathrm{H} 25 \mathrm{~B}$ & 107.9 \\
\hline $\mathrm{O} 2-\mathrm{C} 9-\mathrm{O} 1$ & $123.1(2)$ & $\mathrm{C} 25-\mathrm{C} 26-\mathrm{C} 27$ & $111.6(2)$ \\
\hline $\mathrm{O} 2-\mathrm{C} 9-\mathrm{C} 8$ & $118.9(2)$ & $\mathrm{C} 25-\mathrm{C} 26-\mathrm{H} 26 \mathrm{~A}$ & 109.3 \\
\hline $\mathrm{O} 1-\mathrm{C} 9-\mathrm{C} 8$ & $118.0(2)$ & $\mathrm{C} 27-\mathrm{C} 26-\mathrm{H} 26 \mathrm{~A}$ & 109.3 \\
\hline $\mathrm{C} 11-\mathrm{C} 10-\mathrm{C} 8$ & $113.3(2)$ & $\mathrm{C} 25-\mathrm{C} 26-\mathrm{H} 26 \mathrm{~B}$ & 109.3 \\
\hline $\mathrm{C} 11-\mathrm{C} 10-\mathrm{H} 10 \mathrm{~A}$ & 108.9 & $\mathrm{C} 27-\mathrm{C} 26-\mathrm{H} 26 \mathrm{~B}$ & 109.3 \\
\hline $\mathrm{C} 8-\mathrm{C} 10-\mathrm{H} 10 \mathrm{~A}$ & 108.9 & $\mathrm{H} 26 \mathrm{~A}-\mathrm{C} 26-\mathrm{H} 26 \mathrm{~B}$ & 108.0 \\
\hline $\mathrm{C} 11-\mathrm{C} 10-\mathrm{H} 10 \mathrm{~B}$ & 108.9 & $\mathrm{C} 26-\mathrm{C} 27-\mathrm{C} 28$ & $110.34(19)$ \\
\hline $\mathrm{C} 8-\mathrm{C} 10-\mathrm{H} 10 \mathrm{~B}$ & 108.9 & $\mathrm{C} 26-\mathrm{C} 27-\mathrm{H} 27 \mathrm{~A}$ & 109.6 \\
\hline $\mathrm{H} 10 \mathrm{~A}-\mathrm{C} 10-\mathrm{H} 10 \mathrm{~B}$ & 107.7 & $\mathrm{C} 28-\mathrm{C} 27-\mathrm{H} 27 \mathrm{~A}$ & 109.6 \\
\hline $\mathrm{O} 4-\mathrm{C} 11-\mathrm{O} 3$ & $123.6(2)$ & $\mathrm{C} 26-\mathrm{C} 27-\mathrm{H} 27 \mathrm{~B}$ & 109.6 \\
\hline $\mathrm{O} 4-\mathrm{C} 11-\mathrm{C} 10$ & $123.0(2)$ & $\mathrm{C} 28-\mathrm{C} 27-\mathrm{H} 27 \mathrm{~B}$ & 109.6 \\
\hline $\mathrm{O} 3-\mathrm{C} 11-\mathrm{C} 10$ & $113.3(2)$ & $\mathrm{H} 27 \mathrm{~A}-\mathrm{C} 27-\mathrm{H} 27 \mathrm{~B}$ & 108.1 \\
\hline $\mathrm{C} 13-\mathrm{C} 12-\mathrm{C} 17$ & $110.75(19)$ & $\mathrm{C} 29-\mathrm{C} 28-\mathrm{C} 27$ & $110.96(18)$ \\
\hline $\mathrm{C} 13-\mathrm{C} 12-\mathrm{H} 12 \mathrm{~A}$ & 109.5 & $\mathrm{C} 29-\mathrm{C} 28-\mathrm{C} 23$ & $111.73(19)$ \\
\hline
\end{tabular}




\begin{tabular}{|c|c|c|c|}
\hline $\mathrm{C} 17-\mathrm{C} 12-\mathrm{H} 12 \mathrm{~A}$ & 109.5 & $\mathrm{C} 27-\mathrm{C} 28-\mathrm{C} 23$ & $109.36(18)$ \\
\hline $\mathrm{C} 13-\mathrm{C} 12-\mathrm{H} 12 \mathrm{~B}$ & 109.5 & $\mathrm{C} 29-\mathrm{C} 28-\mathrm{H} 28$ & 108.2 \\
\hline $\mathrm{C} 17-\mathrm{C} 12-\mathrm{H} 12 \mathrm{~B}$ & 109.5 & $\mathrm{C} 27-\mathrm{C} 28-\mathrm{H} 28$ & 108.2 \\
\hline $\mathrm{H} 12 \mathrm{~A}-\mathrm{C} 12-\mathrm{H} 12 \mathrm{~B}$ & 108.1 & $\mathrm{C} 23-\mathrm{C} 28-\mathrm{H} 28$ & 108.2 \\
\hline $\mathrm{C} 14-\mathrm{C} 13-\mathrm{C} 12$ & $111.4(2)$ & $\mathrm{C} 30-\mathrm{C} 29-\mathrm{C} 28$ & $126.6(2)$ \\
\hline $\mathrm{C} 14-\mathrm{C} 13-\mathrm{H} 13 \mathrm{~A}$ & 109.3 & $\mathrm{C} 30-\mathrm{C} 29-\mathrm{H} 29$ & 116.7 \\
\hline $\mathrm{C} 12-\mathrm{C} 13-\mathrm{H} 13 \mathrm{~A}$ & 109.3 & $\mathrm{C} 28-\mathrm{C} 29-\mathrm{H} 29$ & 116.7 \\
\hline $\mathrm{C} 14-\mathrm{C} 13-\mathrm{H} 13 \mathrm{~B}$ & 109.3 & $\mathrm{C} 29-\mathrm{C} 30-\mathrm{C} 31$ & $118.6(2)$ \\
\hline $\mathrm{C} 12-\mathrm{C} 13-\mathrm{H} 13 \mathrm{~B}$ & 109.3 & $\mathrm{C} 29-\mathrm{C} 30-\mathrm{C} 32$ & $124.9(2)$ \\
\hline $\mathrm{H} 13 \mathrm{~A}-\mathrm{C} 13-\mathrm{H} 13 \mathrm{~B}$ & 108.0 & $\mathrm{C} 31-\mathrm{C} 30-\mathrm{C} 32$ & $116.53(19)$ \\
\hline $\mathrm{C} 13-\mathrm{C} 14-\mathrm{C} 15$ & $111.59(18)$ & $\mathrm{O} 9-\mathrm{C} 31-\mathrm{O} 10$ & $123.3(2)$ \\
\hline $\mathrm{C} 13-\mathrm{C} 14-\mathrm{H} 14 \mathrm{~A}$ & 109.3 & $\mathrm{O} 9-\mathrm{C} 31-\mathrm{C} 30$ & $122.18(19)$ \\
\hline $\mathrm{C} 15-\mathrm{C} 14-\mathrm{H} 14 \mathrm{~A}$ & 109.3 & $\mathrm{O} 10-\mathrm{C} 31-\mathrm{C} 30$ & $114.5(2)$ \\
\hline $\mathrm{C} 13-\mathrm{C} 14-\mathrm{H} 14 \mathrm{~B}$ & 109.3 & $\mathrm{C} 30-\mathrm{C} 32-\mathrm{C} 33$ & $112.5(2)$ \\
\hline $\mathrm{C} 15-\mathrm{C} 14-\mathrm{H} 14 \mathrm{~B}$ & 109.3 & $\mathrm{C} 30-\mathrm{C} 32-\mathrm{H} 32 \mathrm{~A}$ & 109.1 \\
\hline $\mathrm{H} 14 \mathrm{~A}-\mathrm{C} 14-\mathrm{H} 14 \mathrm{~B}$ & 108.0 & $\mathrm{C} 33-\mathrm{C} 32-\mathrm{H} 32 \mathrm{~A}$ & 109.1 \\
\hline $\mathrm{C} 14-\mathrm{C} 15-\mathrm{C} 16$ & $111.2(2)$ & $\mathrm{C} 30-\mathrm{C} 32-\mathrm{H} 32 \mathrm{~B}$ & 109.1 \\
\hline $\mathrm{C} 14-\mathrm{C} 15-\mathrm{H} 15 \mathrm{~A}$ & 109.4 & $\mathrm{C} 33-\mathrm{C} 32-\mathrm{H} 32 \mathrm{~B}$ & 109.1 \\
\hline $\mathrm{C} 16-\mathrm{C} 15-\mathrm{H} 15 \mathrm{~A}$ & 109.4 & $\mathrm{H} 32 \mathrm{~A}-\mathrm{C} 32-\mathrm{H} 32 \mathrm{~B}$ & 107.8 \\
\hline $\mathrm{C} 14-\mathrm{C} 15-\mathrm{H} 15 \mathrm{~B}$ & 109.4 & $\mathrm{O} 11-\mathrm{C} 33-\mathrm{O} 12$ & $124.0(2)$ \\
\hline $\mathrm{C} 16-\mathrm{C} 15-\mathrm{H} 15 \mathrm{~B}$ & 109.4 & $\mathrm{O} 11-\mathrm{C} 33-\mathrm{C} 32$ & $123.3(2)$ \\
\hline $\mathrm{H} 15 \mathrm{~A}-\mathrm{C} 15-\mathrm{H} 15 \mathrm{~B}$ & 108.0 & $\mathrm{O} 12-\mathrm{C} 33-\mathrm{C} 32$ & $112.7(2)$ \\
\hline $\mathrm{C} 6-\mathrm{C} 1-\mathrm{C} 2-\mathrm{C} 3$ & $-57.4(3)$ & $\mathrm{C} 17-\mathrm{C} 18-\mathrm{C} 19-\mathrm{C} 20$ & $177.59(19)$ \\
\hline $\mathrm{C} 1-\mathrm{C} 2-\mathrm{C} 3-\mathrm{C} 4$ & $55.7(3)$ & $\mathrm{C} 17-\mathrm{C} 18-\mathrm{C} 19-\mathrm{C} 21$ & $-0.7(4)$ \\
\hline $\mathrm{C} 2-\mathrm{C} 3-\mathrm{C} 4-\mathrm{C} 5$ & $-54.5(3)$ & $\mathrm{C} 18-\mathrm{C} 19-\mathrm{C} 20-\mathrm{O} 6$ & $-176.6(2)$ \\
\hline $\mathrm{C} 3-\mathrm{C} 4-\mathrm{C} 5-\mathrm{C} 6$ & $55.6(3)$ & $\mathrm{C} 21-\mathrm{C} 19-\mathrm{C} 20-\mathrm{O} 6$ & $1.9(3)$ \\
\hline $\mathrm{C} 2-\mathrm{C} 1-\mathrm{C} 6-\mathrm{C} 7$ & $177.96(19)$ & $\mathrm{C} 18-\mathrm{C} 19-\mathrm{C} 20-\mathrm{O} 5$ & $3.4(3)$ \\
\hline $\mathrm{C} 2-\mathrm{C} 1-\mathrm{C} 6-\mathrm{C} 5$ & $56.9(3)$ & $\mathrm{C} 21-\mathrm{C} 19-\mathrm{C} 20-\mathrm{O} 5$ & $-178.14(18)$ \\
\hline $\mathrm{C} 4-\mathrm{C} 5-\mathrm{C} 6-\mathrm{C} 7$ & $-179.48(19)$ & $\mathrm{C} 18-\mathrm{C} 19-\mathrm{C} 21-\mathrm{C} 22$ & $114.2(2)$ \\
\hline $\mathrm{C} 4-\mathrm{C} 5-\mathrm{C} 6-\mathrm{C} 1$ & $-56.6(3)$ & $\mathrm{C} 20-\mathrm{C} 19-\mathrm{C} 21-\mathrm{C} 22$ & $-64.2(2)$ \\
\hline $\mathrm{C} 1-\mathrm{C} 6-\mathrm{C} 7-\mathrm{C} 8$ & $125.9(2)$ & $\mathrm{C} 19-\mathrm{C} 21-\mathrm{C} 22-\mathrm{O} 7$ & $-25.6(3)$ \\
\hline $\mathrm{C} 5-\mathrm{C} 6-\mathrm{C} 7-\mathrm{C} 8$ & $-112.7(3)$ & $\mathrm{C} 19-\mathrm{C} 21-\mathrm{C} 22-\mathrm{O} 8$ & $155.81(18)$ \\
\hline $\mathrm{C} 6-\mathrm{C} 7-\mathrm{C} 8-\mathrm{C} 9$ & $175.5(2)$ & $\mathrm{C} 28-\mathrm{C} 23-\mathrm{C} 24-\mathrm{C} 25$ & $55.4(3)$ \\
\hline $\mathrm{C} 6-\mathrm{C} 7-\mathrm{C} 8-\mathrm{C} 10$ & $-2.1(4)$ & $\mathrm{C} 23-\mathrm{C} 24-\mathrm{C} 25-\mathrm{C} 26$ & $-53.0(3)$ \\
\hline $\mathrm{C} 7-\mathrm{C} 8-\mathrm{C} 9-\mathrm{O} 2$ & $-178.8(2)$ & $\mathrm{C} 24-\mathrm{C} 25-\mathrm{C} 26-\mathrm{C} 27$ & $53.8(3)$ \\
\hline $\mathrm{C} 10-\mathrm{C} 8-\mathrm{C} 9-\mathrm{O} 2$ & $-0.9(3)$ & $\mathrm{C} 25-\mathrm{C} 26-\mathrm{C} 27-\mathrm{C} 28$ & $-56.9(3)$ \\
\hline $\mathrm{C} 7-\mathrm{C} 8-\mathrm{C} 9-\mathrm{O} 1$ & $0.9(3)$ & $\mathrm{C} 26-\mathrm{C} 27-\mathrm{C} 28-\mathrm{C} 29$ & $-177.58(19)$ \\
\hline $\mathrm{C} 10-\mathrm{C} 8-\mathrm{C} 9-\mathrm{O} 1$ & $178.79(19)$ & $\mathrm{C} 26-\mathrm{C} 27-\mathrm{C} 28-\mathrm{C} 23$ & $58.7(3)$ \\
\hline $\mathrm{C} 7-\mathrm{C} 8-\mathrm{C} 10-\mathrm{C} 11$ & $-120.5(2)$ & $\mathrm{C} 24-\mathrm{C} 23-\mathrm{C} 28-\mathrm{C} 29$ & $178.6(2)$ \\
\hline $\mathrm{C} 9-\mathrm{C} 8-\mathrm{C} 10-\mathrm{C} 11$ & $61.8(2)$ & $\mathrm{C} 24-\mathrm{C} 23-\mathrm{C} 28-\mathrm{C} 27$ & $-58.2(3)$ \\
\hline $\mathrm{C} 8-\mathrm{C} 10-\mathrm{C} 11-\mathrm{O} 4$ & $25.3(3)$ & $\mathrm{C} 27-\mathrm{C} 28-\mathrm{C} 29-\mathrm{C} 30$ & $82.5(3)$ \\
\hline $\mathrm{C} 8-\mathrm{C} 10-\mathrm{C} 11-\mathrm{O} 3$ & $-156.38(19)$ & $\mathrm{C} 23-\mathrm{C} 28-\mathrm{C} 29-\mathrm{C} 30$ & $-155.2(2)$ \\
\hline $\mathrm{C} 17-\mathrm{C} 12-\mathrm{C} 13-\mathrm{C} 14$ & $57.2(3)$ & $\mathrm{C} 28-\mathrm{C} 29-\mathrm{C} 30-\mathrm{C} 31$ & $179.0(2)$ \\
\hline $\mathrm{C} 12-\mathrm{C} 13-\mathrm{C} 14-\mathrm{C} 15$ & $-54.9(3)$ & $\mathrm{C} 28-\mathrm{C} 29-\mathrm{C} 30-\mathrm{C} 32$ & $0.1(4)$ \\
\hline $\mathrm{C} 13-\mathrm{C} 14-\mathrm{C} 15-\mathrm{C} 16$ & $53.7(3)$ & $\mathrm{C} 29-\mathrm{C} 30-\mathrm{C} 31-\mathrm{O} 9$ & $9.4(3)$ \\
\hline $\mathrm{C} 14-\mathrm{C} 15-\mathrm{C} 16-\mathrm{C} 17$ & $-55.4(3)$ & $\mathrm{C} 32-\mathrm{C} 30-\mathrm{C} 31-\mathrm{O} 9$ & $-171.6(2)$ \\
\hline $\mathrm{C} 15-\mathrm{C} 16-\mathrm{C} 17-\mathrm{C} 18$ & $-179.20(19)$ & $\mathrm{C} 29-\mathrm{C} 30-\mathrm{C} 31-\mathrm{O} 10$ & $-169.4(2)$ \\
\hline
\end{tabular}




$\begin{array}{llll}\mathrm{C} 15-\mathrm{C} 16-\mathrm{C} 17-\mathrm{C} 12 & 57.1(3) & \mathrm{C} 32-\mathrm{C} 30-\mathrm{C} 31-\mathrm{O} 10 & 9.7(3) \\ \mathrm{C} 13-\mathrm{C} 12-\mathrm{C} 17-\mathrm{C} 18 & 178.21(19) & \mathrm{C} 29-\mathrm{C} 30-\mathrm{C} 32-\mathrm{C} 33 & -120.9(2) \\ \mathrm{C} 13-\mathrm{C} 12-\mathrm{C} 17-\mathrm{C} 16 & -57.6(3) & \mathrm{C} 31-\mathrm{C} 30-\mathrm{C} 32-\mathrm{C} 33 & 60.1(2) \\ \mathrm{C} 16-\mathrm{C} 17-\mathrm{C} 18-\mathrm{C} 19 & 160.7(2) & \mathrm{C} 30-\mathrm{C} 32-\mathrm{C} 33-\mathrm{O} 11 & 15.5(3) \\ \mathrm{C} 12-\mathrm{C} 17-\mathrm{C} 18-\mathrm{C} 19 & -76.6(3) & \mathrm{C} 30-\mathrm{C} 32-\mathrm{C} 33-\mathrm{O} 12 & -165.74(17)\end{array}$

Hydrogen-bond geometry $\left(\AA,{ }^{\circ}\right)$

\begin{tabular}{lllll}
\hline$D-\mathrm{H} \cdots A$ & $D-\mathrm{H}$ & $\mathrm{H} \cdots A$ & $D \cdots A$ & $D-\mathrm{H} \cdots A$ \\
\hline $\mathrm{O} 12-\mathrm{H} 12 \cdots \mathrm{O} 11^{\mathrm{i}}$ & $0.87(2)$ & $1.78(2)$ & $2.656(2)$ & $175(3)$ \\
$\mathrm{O} 10-\mathrm{H} 10 \cdots \mathrm{O} 9^{\mathrm{ii}}$ & $0.95(2)$ & $1.63(2)$ & $2.586(2)$ & $175(3)$ \\
$\mathrm{O} 8-\mathrm{H} 8 \cdots \mathrm{O} 44^{\mathrm{iii}}$ & $0.95(3)$ & $1.70(3)$ & $2.642(2)$ & $172(3)$ \\
$\mathrm{O} 5-\mathrm{H} 5 \cdots \mathrm{O} 2$ & $0.92(2)$ & $1.68(2)$ & $2.594(2)$ & $175(3)$ \\
$\mathrm{O} 3-\mathrm{H} 3 \cdots \mathrm{O} 7^{\mathrm{iv}}$ & $0.90(2)$ & $1.75(2)$ & $2.644(2)$ & $173(3)$ \\
$\mathrm{O} 1-\mathrm{H} 1 \cdots \mathrm{O} 6$ & $0.93(2)$ & $1.66(2)$ & $2.591(2)$ & $175(3)$
\end{tabular}

Symmetry codes: (i) $-x+1,-y+1,-z+2$; (ii) $-x,-y+1,-z+2$; (iii) $x-1, y, z$; (iv) $x+1, y, z$. 